Article:

Driving flows in microfluidic paper-based analytical devices with a cholinium based poly(ionic liquid) hydrogel

Tuğçe Akyazi, Alexandru Tudor, Dermot Diamond, Lourdes BasabeDesmonts, Larisa Florea, Fernando Benito-Lopez

Sensors and Actuators B: Chemical $261: 372-378$ (2018)

This work is made available online in accordance with publisher policies. To see the final version of this work please visit the publisher's website. Access to the published online version may require a subscription.

Link to publisher's version:

http://doi.org/10.1016/j.snb.2018.01.154

Copyright statement: (c) 2018 Elsevier Ltd. Full-text reproduced in accordance with the publisher's self-archiving policy.

This manuscript version is made available under the CC-BY-NC-ND 4.0 license http://creativecommons.org/licenses/by-nc-nd/4.0

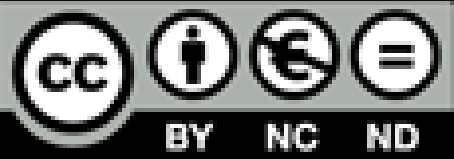




\title{
DRIVING FLOWS IN MICROFLUIDIC PAPER-BASED ANALYTICAL DEVICES WITH A CHOLINIUM BASED POLY IONIC LIQUID HYDROGEL
}

\author{
Tuğçe Akyazi ${ }^{1,2, \#, ~ A l e x a n d r u ~ T u d o r ~}{ }^{3, \#}$, Dermot Diamond ${ }^{3}$, Lourdes Basabe-Desmonts ${ }^{4,5}$, \\ Larisa Florea ${ }^{3,}$, Fernando Benito-Lopez ${ }^{1, *}$ \\ ${ }^{1}$ Analytical Microsystems \& Materials for Lab-on-a-Chip (AMMa-LOAC) Group, Microfluidics \\ Cluster UPV/EHU, Analytical Chemistry Department, University of the Basque Country \\ UPV/EHU, Vitoria-Gasteiz, Spain, ${ }^{2}$ University of Navarra, TECNUN, SPAIN, ${ }^{3}$ Insight Centre for \\ Data Analytics, National Centre for Sensor Research, Dublin City University, IRELAND, \\ ${ }^{4}$ BIOMICs Research Group, Lascaray Ikergunea, Research Center, University of the Basque \\ Country, SPAIN, ${ }^{5}$ Ikerbasque, Basque Foundation for Science, SPAIN. "Both authors contributed \\ equally to this work
}

\begin{abstract}
Paper microfluidics technology requires effective handling and control of fluids, and this remains as a significant obstacle for their accessibility by end-users, inhibiting their transition from the laboratory into the market. Here, the synthesis, characterisation and performance as negative passive pumps of cholinium based poly(ionic liquid) hydrogels are presented, for the manipulation of fluid flow in microfluidic paper-based analytical devices. The hydrogel was able to direct the fluid flow preferentially, and prevent fluid from flowing in other directions, thus opening possibilities for effective fluid flow manipulation in $\mu$ PADs. Moreover, the hydrogel 'passive pump' improves the water retention capacity of the $\mu$ PAD by a factor of nine when compared to the bare $\mu \mathrm{PAD}$. Furthermore, the operational lifetime of this $\mu \mathrm{PAD}$ configuration was greatly increased by the integration of the hydrogel passive pumps.
\end{abstract}

Keywords: Paper microfluidics, passive pump, poly(ionic liquid), paper microfluidics, $\mu$ PADs, hydrogel. 


\section{Introduction}

The field of "Lab-on-a-Chip" (LOC) has an inherently interdisciplinary nature that requires input from widely differing knowledge domains to develop novel analytical platforms. The greatest potential of these systems is the integration of multiple functional elements into a small platform to generate truly sample-in/answer-out systems [1]. The design, fabrication, fluid control, sample handling, integration and analysis techniques are under continuous development and significant research is still needed to improve the capabilities of these platforms [2].

The critical need of a large variety of high performance components for fluid control and transport such as mixers, actuators, separators, valves and pumps leads to high production costs for these microfluidic devices. Therefore, despite the significant advances achieved in the microfluidics field, the number of commercially available products based on microfluidics devices and components remains quite low regardless of few exceptions like the home pregnancy test kit, microarrays and some medical diagnostics devices with very particular applications [3]. In other words, the increase in production cost of a device results in the decrease of their market adoption possibilities, where penetration of microfluidics into applications with significant socio-economic impact has been restricted almost entirely to single use devices (disposables) rather than applications that require continuous long-term monitoring approaches.

Consequently, "Lab on a paper" has been developed to provide an answer to the need of simple, cheap and autonomous devices, which could be able to reach the end users easily [4]. They exhibit not only most of the same properties as classical microfluidics, but also the strength of a wellfocused commercialisation path [5]. Paper is considered as a highly attractive and promising substrate material for microfluidics due to its extremely low cost and ubiquity, as well as its great mechanical properties comprising flexibility, lightness, variable thickness, and liquid transport and separation capabilities [4]. Microfluidic paper-based analytical devices ( $\mu$ PADs) are a new group of analytical instruments that demonstrate an innovative low-cost platform technology for fluid handling and analysis, providing simple fabrication and operation, thus enabling a wide range of applications. For instance, the possibility of analysing complex biochemical samples within one analytical run was recently demonstrated with the incorporation of several fluidic operations like transportation, sorting, mixing and separation, performed in the device [6].

Paper is made of cellulose fibres, which are the driving force for the wicking of fluids by capillary action. Therefore, there is no need for external pumps to provide fluid transport through the paper, 
unlike the traditional microfluidic devices. However, this advantage comes with a drawback. Isotropic wicking behaviour of paper and fluid transportation by any exposed surface area makes accurate control of the fluid transport together with flow control to be highly challenging and complicated $[7,8]$. As a result, $\mu$ PADs need ways to implement effective manipulation and control of fluid behaviour through paper. Because of this, significant obstacles are generated in the reproducibility during device fabrication, and performance, precluding their production in large volumes. Hence, fluid control in paper microfluidic devices is currently one of the main investigation paths for researchers interested in developing new capabilities in $\mu$ PADs.

In order to achieve better flow control in microfluidic paper devices, several patterning processes such as photolithography [9], ink jet printing [10] and wax printing [11], using hydrophobic materials, were adopted. They were developed as the first step to generate operative $\mu$ PADs but they are not sufficiently proficient to provide proper fluidic control in the device. Therefore, fluidic switches, microvalves, timers and micropumps integrated into $\mu$ PADs can provide fluid control in the channels, as well as minimising dead volumes. The first microfluidic switch in a $\mu$ PAD was introduced by Li et al. [12] by applying pressure, manually, to allow or block the fluid flow. Later, Whitesides' group developed a pressure sensitive valve with a more complex mechanism in a three dimensional paper based microfluidic device [13]. Fu and her team [14] demonstrated that the time to deliver multiple fluids can be partially controlled and modified by designing different path lengths from each inlet and by drop-casting different fluid volumes to each inlet. Later, Houghtaling et al. [15] developed an innovative shut-off valve in the form of a dissolvable bridge structure enabling self-delivery of different fluid volumes from a common inlet to different pathways in a paper-based device. Other dissolvable fluidic restrictors, made of sugar, were introduced by Lutz el al. [16] and by Jahanshahi-Anbuhi et al. [17] using a dissolving polymer, the main disadvantage of these dissolvable restrictors is that the sample gets polluted and so the chemical and physical characteristics of the sample are modified. More recently, Toley et al. [18] developed a toolkit of paper microfluidic valves using movable paper strips and fluid-triggered expanding elements.

In this regard, stimuli-responsive gels are receiving considerable attention in microfluidic devices, due to their autonomous response towards changes in their local environment $[19,20]$. They are considered smart materials since they are able to carry out functions by weak changes in their surroundings without the need of any human intervention [21]. Owing to their physical or chemical 
characteristics, these gels can endure controlled and reversible shape changes in response to external stimuli (magnetic, electric field, temperature, light, $\mathrm{pH}$, solvent composition, etc.) [2224].

Smart hydrogels consist of three-dimensional hydrophilic polymer networks capable of absorbing or releasing large amount of water in response to an external stimulus, mainly $\mathrm{pH}$, light or temperature, generating an abrupt change in their volume. When the stimulus is removed, the gel returns back to its original configuration, which means that the volume change is usually reversible [25]. These properties have inspired scientists to integrate hydrogels within microfluidic systems in order to achieve fluidic control and manipulation [26-31]. In paper deceives, little work has been carried out on the integration of these type of materials for fluid control. For instance, Niedl et al. [19] made the use of stored chemicals possible by utilising responsive hydrogels as fluid reservoirs in $\mu$ PADs. The controlled release of the hydrogel fluid was achieved by an external stimulus, temperature, enabling multi-step sequences of chemical reaction on a $\mu$ PAD. Another example of the use of hydrogels on a $\mu$ PAD to manipulate the fluidic flow is the study of Yang et al. [20]. They used target-responsive hydrogels to mediate fluidic flow and signal readout in a paper-based point of care assay designed for simultaneous detection of multiple targets.

Recently, ionogels have been used within microfluidic devices as actuators [32-35]. They are a new class of stimuli-responsive polymer gels with an ionic liquid (IL) within their polymer matrixes where the ionic liquid can enhance the mechanical strength and the physical robustness of the gel [34-36]. Moreover, the possibility of tuning the chemical and physical properties of the ionogel by changing the ILs enables precise control of the actuation in the microfluidic devices [2, 37]. In our previous work, we integrated ionogels into $\mu$ PADs for fluidic flow manipulation as passive pumps [38] and later as negative passive pumps to drive the liquid flow towards the direction of the pump [39]. These types of configurations open the possibility to detect multiple analytes sequentially by circumventing the main $\mu$ PAD channel's wicking force, without the need to design complicated device configurations.

Despite the great potential of ionogels as smart materials for fluid control in $\mu$ PADs, their performance is not very good for long term and continuous operation, therefore other materials could also improve $\mu$ PAD performance, such as superabsorbent polymers, namely superabsorbent poly(ionic liquid)s (PILs). PILs are ILs that features a polymerisable group in the cation, the anion, or both. By synthesising PILs, the typical properties of ILs are transferred to the polymeric 
material, including high chemical and thermal stability, ionic conductivity, and highly tunable structures [40-44].

Here, we report the synthesis and thermal characterisation of a novel cholinium based crosslinked poly(ionic liquid) hydrogel, the integration of the hydrogel in a $\mu$ PAD and the performance of the hydrogel as a negative passive pump to control liquid flow and storage of fluids in the $\mu$ PAD.

\section{Materials and Methods}

\subsection{Materials and reagents}

Choline chloride $\geq 98 \%$ (ChoCl, BioReagent, suitable for cell culture, suitable for insect cell culture), Potassium 3-sulfopropyl acrylate (KSPA), polyethylene glycol diacrylate $\left(\mathrm{M}_{\mathrm{w}} \sim 320,100\right.$ ppm MEHQ as inhibitor) (PEG250), 2-Hydroxy-2-methylpropiophenone 97\% (HMPP), ethanol $\left(\right.$ Chormasolv $^{\circledR}$, HPLC grade, $\geq 99.8 \%$ ) were bought from Sigma Aldrich ${ }^{\circledR}$ and used as received. Dimethyl Sulfoxide (DMSO) was HPLC grade and used as received. Deionised water (18.2 $\mathrm{M} \Omega \cdot \mathrm{cm}^{-1}$ ) (DI water) was purified using a Merck Millipore Milli-Q Water Purification System.

For the visual monitoring of the liquid flow, red, yellow and blue food dyes (McCormick, Sabadell, Spain) were used.

\section{$2.2 \mu \mathrm{PAD}$ fabrication}

Whatman Filter paper Grade 1, wax printer XEROX ColourQube8580 and a hot plate (Labnet International Inc., USA) were used in order to fabricate the $\mu$ PADs. The design of the devices was carried out with the software application AutoCAD ${ }^{\mathrm{TM}}$. Adhesive Research, Ireland, generously provided the pressure sensitive adhesive layers. The $\mu$ PAD consists of a single channel $(32 \mathrm{~mm}$ length and $1.5 \mathrm{~mm}$ width) with the inlet at the middle of the channel (6 mm radius dimension) and two outlets (10 $\mathrm{mm}$ radius dimension) situated at the same distance from the inlet.

\subsection{Synthesis of ChoSPA ionic liquid monomer}

Cholinium sulfopropyl acrylate (ChoSPA) was synthesised by dissolving $7.5 \mathrm{~g}$ of $\mathrm{ChoCl}$ together with $16.2 \mathrm{~g}$ of KSPA (1.3 molar equivalents) in $20 \mathrm{~mL}$ of DI water (Scheme SI-1). The reaction mixture was magnetically stirred at room temperature for $72 \mathrm{~h}$. Following this, it was poured into a beaker and moved to a vacuum oven, which was kept at $45^{\circ} \mathrm{C}$ and 200 mbar for $32 \mathrm{~h}$. This 
resulted in a crystalline deposit forming at the bottom of the beaker. To finalise the crystallisation process, the beaker was kept for further $48 \mathrm{~h}$ in a vacuum oven under normal conditions of temperature and pressure. The resulting ChoSPA crystalline product was extracted using absolute ethanol, which was poured into the beaker, followed by magnetic stirring and gravity filtration of the resulting heterogeneous mixture. The extracted solution was concentrated by rotary evaporation at $40^{\circ} \mathrm{C}$, followed by complete overnight drying using a high vacuum line (0.5 mbar). The resulting viscous, translucent white coloured product was obtained with a yield of $\sim 60 \%$. ChoSPA - ${ }^{1} \mathrm{H}-\mathrm{NMR} \delta_{\mathrm{H}}(400 \mathrm{MHz}): 1.98-2.05\left(\mathrm{~m}, 2 \mathrm{H}, \mathrm{CH}_{2}\right), 2.88-2.92$ (p, 2H, CH ), 3.08 (s, 9H, $\mathrm{CH}_{3}$ ), 3.38-3.41 (p, 2H, $\left.\mathrm{CH}_{2}\right), 3.91-3.95\left(\mathrm{~m}, 2 \mathrm{H}, \mathrm{CH}_{2}\right), 4.15-4.19$ (t, 2H, $\left.\mathrm{CH}_{2}\right), 5.84-5.87$ (dd, 1H, CH), 6.05-6.12 (dd, 1H, CH), 6.29-6.34 (dd, 1H, CH).

\subsection{Synthesis of the cholinium PIL hydrogel discs for swelling and thermal characterisation}

For the fabrication of ChoSPA hydrogel disks, $0.238 \mathrm{~g}$ of ChoSPA $(800 \mu \mathrm{mol})$ were mixed with $0.238 \mathrm{~g}$ of DMSO:DI water 1:1 mixture, $3.2 \mu \mathrm{L}$ of HMPP $(20 \mu \mathrm{mol})$ and $6 \mu \mathrm{L}$ of PEG250 (16 $\mu \mathrm{mol})$, respectively. The mixture was mechanically stirred until fully dissolved. Following this, $5 \mu \mathrm{L}$ aliquots of monomer mixture were pipetted in circular poly(dimethylsiloxane) moulds (Sylgrad ${ }^{\circledR}-184$, Ireland) with a diameter of $3 \mathrm{~mm}$ and a depth of $1 \mathrm{~mm}$. The polymerisation was done in a UVP CL-1000 Ultraviolet Crosslinker curing chamber using a wavelength of $365 \mathrm{~nm}$ at a power level of $3.5 \mathrm{~mW} \mathrm{~cm}^{-2}$ for $30 \mathrm{~min}$.

\subsection{Swelling studies}

Equilibrium swelling studies were carried out at room temperature. The freshly polymerised hydrogels were firstly weight and then immersed in DI water for swelling. The hydrogel was then removed from the swelling medium at $30 \mathrm{~s}$ time intervals and weighed after all excessive solution on the surface was blotted. The equilibrium swelling ratio (SR) was calculated according to the following equation (eq. 1):

$$
S R=\frac{W s-W i}{W i}
$$

where $w_{s}$ is the weight of the swollen hydrogel at time $t$ and $w_{i}$ is the initial weight of the polymerized gel. Data presented was the mean values of triplicate measurements. 
A single exponential model (eq. 2) was used to determine the swelling rate constant for the ChoSPA hydrogels:

$$
y=a\left(1-e^{-k t}\right)+b
$$

where $y$ is the swelling ratio, $a$ is a scaling factor, $k$ is the first order swelling rate constant $\left(\mathrm{s}^{-1}\right), b$ is the baseline offset, and $t$ is time (s).

\subsection{Thermal characterisation of the hydrogel}

The thermogravimetric analysis was made using a TA Instruments Q50 Thermogravimetric Analysis instrument. The temperature program used consisted of a temperature ramp between room temperature and $500{ }^{\circ} \mathrm{C}$ in $10{ }^{\circ} \mathrm{C} \mathrm{min}^{-1}$. The measurement was done in a $\mathrm{N}_{2}$ atmosphere at a flow rate of $50 \mathrm{~mL} \mathrm{~min}{ }^{-1}$. This ensured that oxidation events did not occur during the decomposition phase.

\section{Results and Discussion}

\subsection{ChoSPA hydrogel characterisation}

The cholinium PIL hydrogel was synthesised following the protocol described in the experimental section. Using the cholinium sulfopropyl acrylate ionic liquid monomer (ChoSPA) and polyethylene glycol diacrylate (PEG250), a crosslinked polymer network was generated; see Figure 1(a). The hydrogel was photopolymerised in $3 \mathrm{~mm}$ disc shapes (Figure 1(b)) or as $500 \mu \mathrm{m}$ thin films (Video S1) that can absorb large quantities of water. The resulting hydrogel discs are shown in Figure 1(b) before swelling (1), after reaching equilibrium swelling in DI water (2) and after complete dehydration at room temperature (3). 

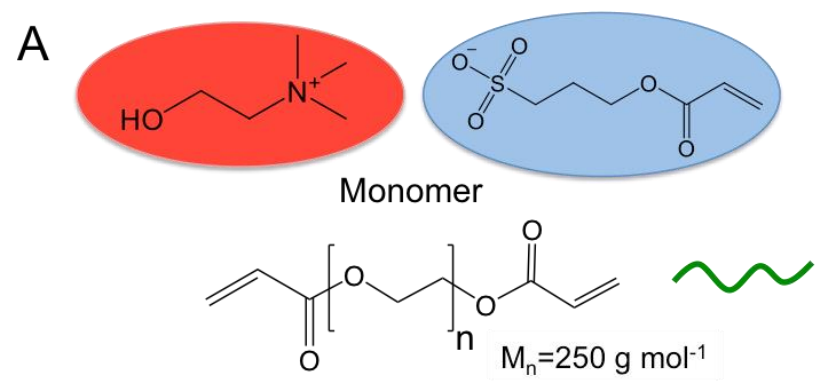

Crosslinker
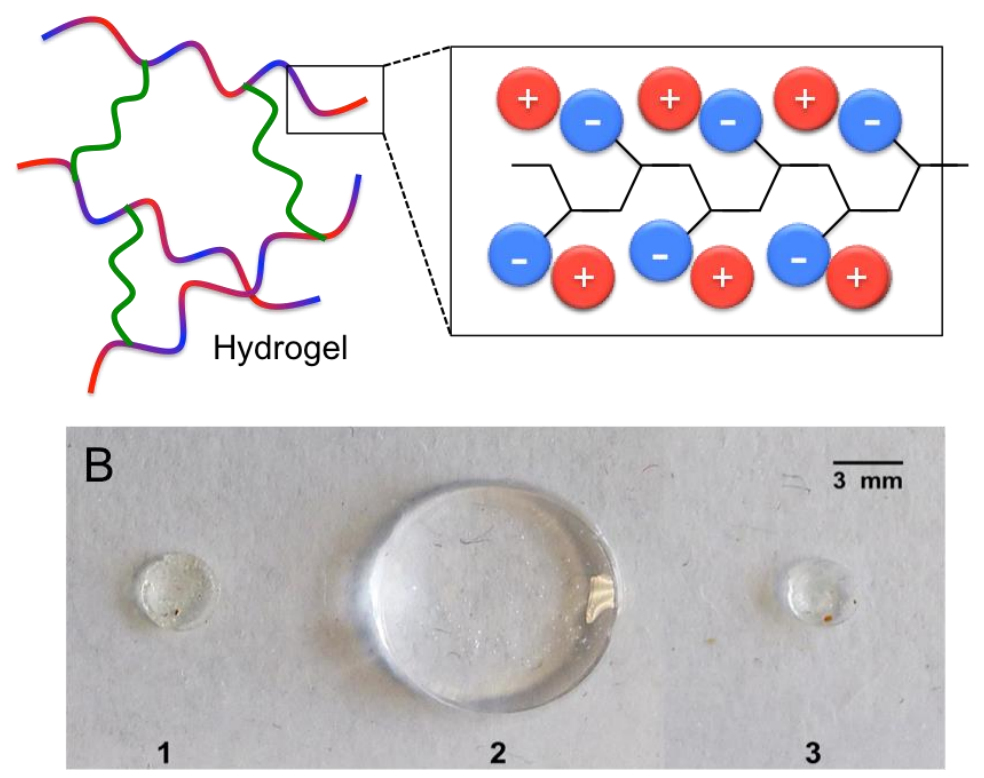

Figure 1. (A) Structure of the different hydrogel chemicals: ChoSPA (top), poly(ethylene glycol) diacrylate (middle) and ChoSPA hydrogel (bottom); (B) Comparison between the sizes of the ChoSPA hydrogel before swelling (1), swelling in DI water for 2 h (2) and after dehydration by drying at room temperature (3).

The equilibrium swelling ratio $(\mathrm{SR}), \mathrm{SR}=11.5 \pm 0.4(\mathrm{n}=3)$, was reached in $\sim 500 \mathrm{~s}$, Figure 2 , and was calculated using Eq. 1. The first order swelling rate constants were estimated by fitting the SR values using Microsoft Excel Solver and eq. 2. The swelling rate constant $(k)$ was found to be $6.17 \times 10^{-3} \mathrm{~s}^{-1}$. The equilibrium swelling ratio and swelling kinetics are important parameters to be considered for the envisaged application of this hydrogel as passive pump in $\mu$ PADs. 


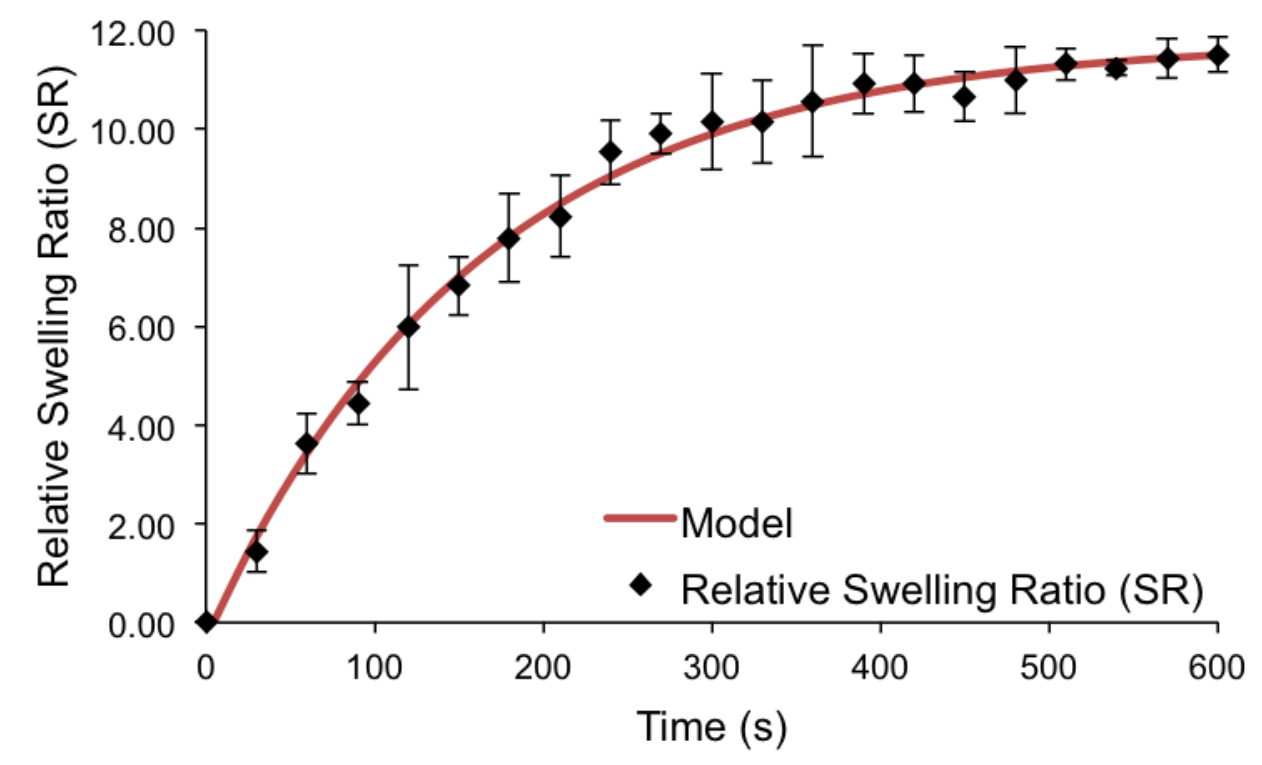

Figure 2. Experimental and fitted swelling kinetic curves for the ChoSPA hydrogels in DI water. Error bars represent the standard deviation $(n=3)$.

Thermal characterisation of the hydrogel was performed using thermogravimetric analysis to compare the thermogravimetric characteristics of the ChoSPA hydrogel before swelling (1), swelling in DI water for $2 \mathrm{~h}(2)$ and after dehydration by drying at room temperature (3). For hydrogel (1) (Figure 1), results showed the amount of water still present inside the hydrogel immediately after polymerisation and the decomposition temperature, respectively. Hydrogel 1 started eliminating water/solvent as soon as the temperature increased. This trend continued up to $\sim 200{ }^{\circ} \mathrm{C}$, when all the water was evaporated, corresponding to a weight loss of $13.0 \%$ compared to the original weight. At this point, the weight of the hydrogel started plateauing until $\sim 280{ }^{\circ} \mathrm{C}$ when it started dropping again, due to the start of the decomposition phase of the hydrogel. The onset temperature of this process is $351{ }^{\circ} \mathrm{C}$ and it continued at the same rate up to $\sim 375{ }^{\circ} \mathrm{C}$, where it started slowing down until $500{ }^{\circ} \mathrm{C}$. At this point, a further $66.8 \%$ of the initial weight was lost. This left hydrogel (1) at $\sim 20.0 \%$ of its original weight. This represents the non-decomposed residue of hydrogel (1) at $500{ }^{\circ} \mathrm{C}$; see Figure S1. Hydrogel (2) (Figure 1), after complete swelling in DI water for $2 \mathrm{~h}$, presented similar behaviour to hydrogel 1, with differences appearing in the magnitude of the heating-induced weight loss events (Figure S2). The weight loss due to water evaporation started immediately after temperature increased, up to $~ 160{ }^{\circ} \mathrm{C}$. At this point $96.9 \%$ 
of the initial mass of the hydrogel was lost. From this temperature up to $\sim 320{ }^{\circ} \mathrm{C}$ the hydrogel mass did not undergo any notable change. At $\sim 340{ }^{\circ} \mathrm{C}$ the decomposition phase began. During this process, the hydrogel mass dropped by $2.6 \%$ of its initial weight until a temperature of $\sim 390$ ${ }^{\circ} \mathrm{C}$ was reached, after which the mass reached a plateau. This signalled the end of the decomposition phase. From Figure S3 it can be observed that hydrogel (3) (Figure 1) presented a similar thermogravimetric analysis curve to hydrogel (1). The weight loss started just after the temperature started increasing and it stopped when the temperature was at $\sim 200{ }^{\circ} \mathrm{C}$. The weight loss during this phase was $14.5 \%$. Following this, there was negligible weight loss until $\sim 280{ }^{\circ} \mathrm{C}$, at which point the decomposition phase started. The onset temperature for this process was 349 ${ }^{\circ} \mathrm{C}$, which was very close to the onset temperature observed in 1 and 2 . During this phase, the hydrogel lost $69.0 \%$ of its weight. This process started slowing down after $\sim 375{ }^{\circ} \mathrm{C}$, but continued until $\sim 500{ }^{\circ} \mathrm{C}$ at which point it reached a plateau. As in the case of hydrogel (1), $20.0 \%$ of the initial weight of the hydrogel was left, representing the non-decomposed part of the hydrogel. In all cases, the hydrogels started losing water as soon as the temperature rose above room temperature. Moreover, their decomposition onset temperatures were very similar at $~ 350{ }^{\circ} \mathrm{C}$. Based on this, it can be concluded that in all three cases, the freshly polymerised, hydrated and dehydrated hydrogels essentially retain their thermogravimetric characteristics. Additionally, the hydrogels retain their hydration capabilities after dehydration.

\subsection{ChoSPA hydrogel as passive pump in $\mu$ PADs}

After thermal characterisation of the hydrogel, the $\mu$ PADs were fabricated using the wax printing method on standard laboratory filter paper. The wax printing method for $\mu$ PAD fabrication is based on (1) designing the shape of the device, (2) patterning hydrophobic wax barriers on the paper surface using a commercially available printer and (3) penetration of wax through all the paper thickness by heating the device on a hot plate to form a complete hydrophobic barrier. The final dimensions of the microfluidic structure, borders and flow channels, are addressed only after a post-heating process [39], in this case $125^{\circ} \mathrm{C}$ for $7 \mathrm{~min}$. The post-heating treatment ensures that there is very little variation in dimensions among $\mu$ PADs. The hydrogel was photopolymerised in a defined disc format over an $80 \mu \mathrm{m}$ diameter pressure sensitive adhesive (PSA) layer. This configuration ensures the hydrogel can be neatly fixed on top of one of the two $\mu$ PAD outlets as 
shown in Figure 3A. Then, the fluid flow behaviour towards the outlets (with hydrogel: outlet-1 and without hydrogel: outlet-2) was compared. Finally, the back of the $\mu$ PAD was covered with another layer of PSA to protect the $\mu \mathrm{PAD}$ and to diminish the effect of solvent evaporation over time during testing. Moreover, this sandwich configuration improved the robustness of the entire device, see Figure 3B.

\section{A Opening for the inlet}

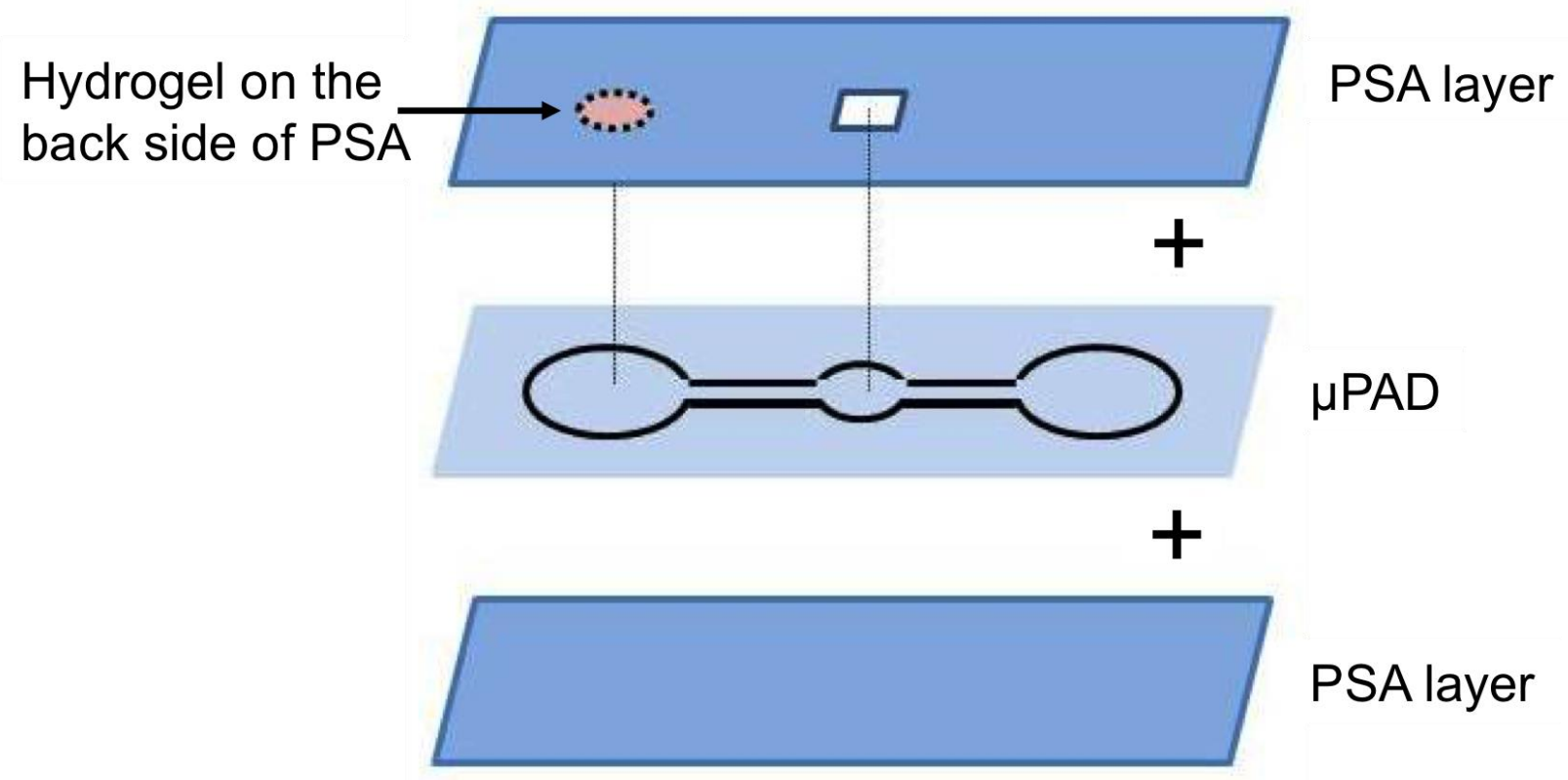

B

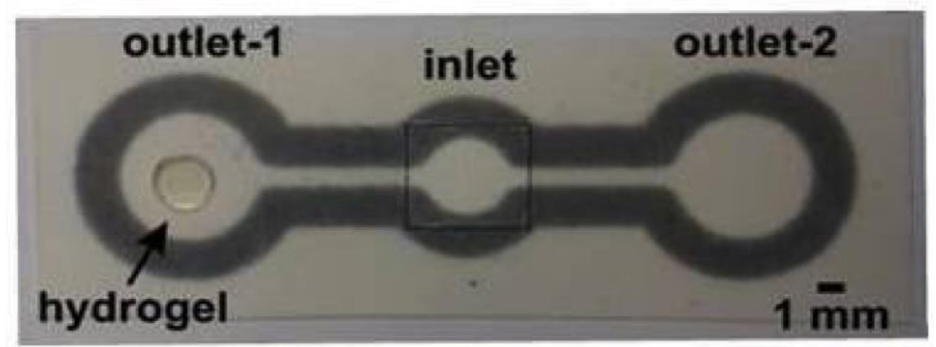

Figure 3. (A) Scheme of the different layers forming the $\mu P A D$. (B) Picture of the $\mu P A D$ after integration of the cholinium PIL hydrogel pump.

Different amounts of cholinium PIL hydrogel $(5,10,15,20,25,30 \mu \mathrm{L})$ were photopolymerised in a disc shape form and placed in the $\mu$ PADs at outlet-1. It was found that $20 \mu \mathrm{L}$ of monomer mixture was the maximum that could be applied, as higher amounts damaged the microfluidic device due 
to bending of the paper at the outlet during the hydration process. However, this is true only for this configuration, as other $\mu \mathrm{PAD}$ configurations may be able to hold higher amounts of hydrogel and so promote longer use models. Therefore, $20 \mu \mathrm{L}$ of cholinium PIL monomer mixture was chosen to investigate the performance of the hydrogel as a passive pump in $\mu$ PADs.

Figure 4A shows the $\mu \mathrm{PAD}$ when a $20 \mu \mathrm{L}$ cholinium PIL hydrogel disc $(1.8 \pm 0.3 \mathrm{~mm}$ diameter by $1.0 \pm 0.4 \mathrm{~mm}$ height) was integrated in the device. In Figure $4 \mathrm{~B}, 50 \mu \mathrm{L}$ of a yellow dye solution was dropped at the inlet of the $\mu \mathrm{PAD}$. The yellow solution flowed through both channels equally, until both outlets got fully wetted. This process occurred at the same time, as can be seen in Figure $4 C$. In a conventional $\mu$ PAD, when all the channels are hydrated, the wicking property of paper no longer has any effect and the device stops working. In this case, the hydrogel passive pump dominates the capillary forces of the paper and all liquid added to the $\mu$ PAD moves towards the hydrogel, in preference to outlet-2.

In order to investigate hydrogel passive pumping capacity of the gel, $60 \mu \mathrm{L}$ of a red dye solution, Figure 4D, was added to the inlet. The liquid flows almost exclusively towards the hydrogel at outlet-1. A small amount of red dye is visible in the channel going to the outlet-2, but no liquid flow was observed. The reason for this behaviour is slow diffusion of dye molecules through the already hydrated paper fibres. In fact no flow was observed towards outlet-2, even after $10 \mathrm{~min}$, as can be seen in Figure 4E and 3F. Conversely, the vast majority of the red dye solution is been absorbed by the hydrogel at outlet-1 (Figure 4G). Subsequently, $70 \mu \mathrm{L}$ of a blue dye solution were added the inlet of the $\mu \mathrm{PAD}$, in several aliquots of $10 \mu \mathrm{L}$. The hydrogel continues pulling the liquid flow towards outlet-1 and accumulating the solution (Figure $4 \mathrm{H}$ ). The final $2 \mu \mathrm{L}$ of the blue solution were not absorbed by the hydrogel, as at this point, the hydrogel had reached its full wicking capacity in this configuration, as it can be seen in Figure 4I.

In both of the above cases, the dye was continuously drawn towards the outlet containing the hydrogel, due to the cholinium PIL hydrogel hydration capacity. The hydrogel was therefore able to direct the fluid flow preferentially towards one of the $\mu$ PAD outlets, and prevent fluid flow to the other outlet. This clearly opens the way for fluid flow manipulation and retention in $\mu$ PADs. This hydrogel 'passive pump' improve the water retention capacity of the $\mu$ PAD by a factor of nine, compared to the bare $\mu \mathrm{PAD}(178 \mu \mathrm{L}$ of liquid), for just $20 \mu \mathrm{L}$ of hydrogel monomer mixture. Obviously, the water retention capacity, and the flow rate, can be increased or reduced by changing the amount of the hydrogel used and the configuration of the $\mu$ PAD. Moreover, the operational 
lifetime of this $\mu \mathrm{PAD}$ configuration, in the present experimental conditions, was over $90 \mathrm{~min}$ of continuous use before reaching saturation of the gel.
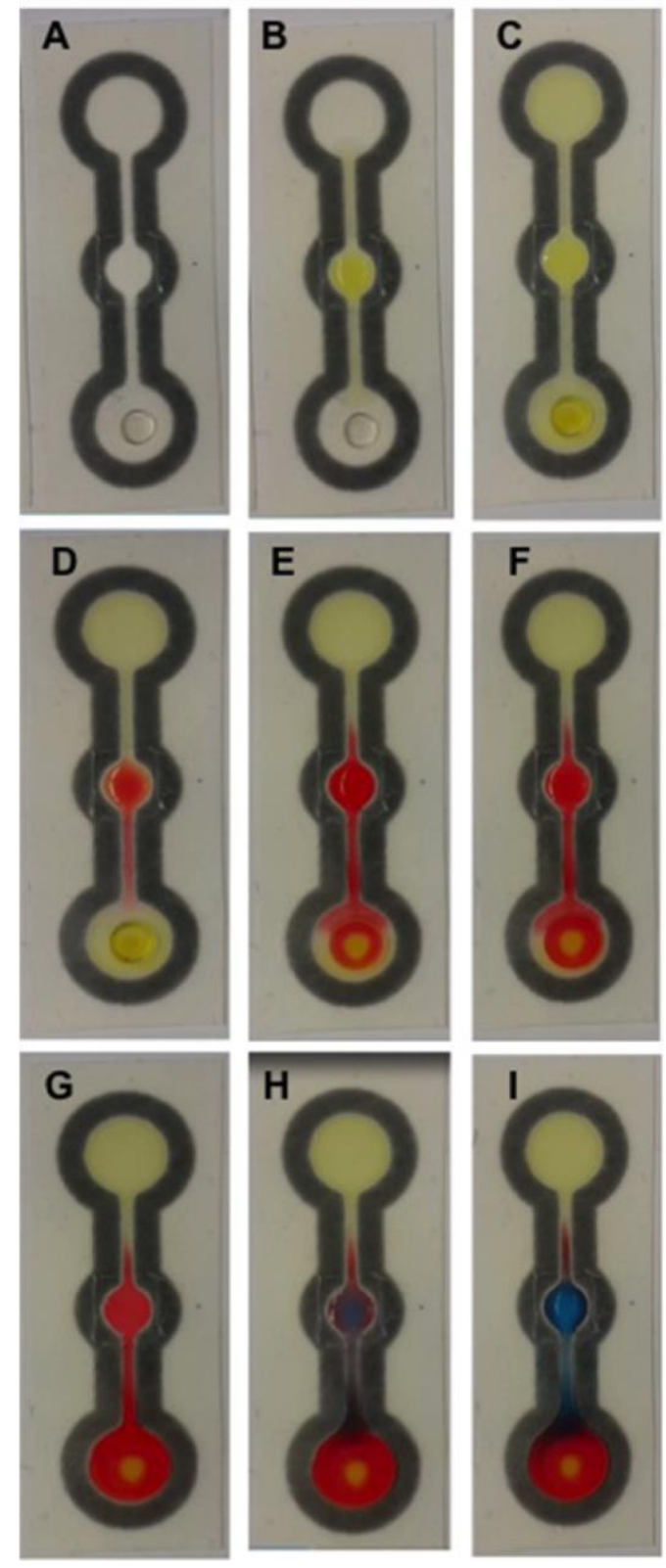

Figure 4. Set of pictures of the $\mu P A D$ over time, while adding different volumes of coloured solutions at $O s$ (A), at $2.5 \mathrm{~min}(B)$, at $25.0 \mathrm{~min}(C)$, at $55.0 \mathrm{~min}(D)$, at $60.0 \mathrm{~min}(E)$, at $65.0 \mathrm{~min}$ $(F)$, at $70.0 \mathrm{~min}(G)$, at $75.0 \mathrm{~min}(\mathrm{H})$, at $90.0 \mathrm{~min}(\mathrm{I})$, after injection of $50 \mu \mathrm{L}$ (yellow dye), followed by $60 \mu \mathrm{L}$ (red dye) and $70 \mu \mathrm{L}$ (blue dye) solutions, at the inlet, in aliquots of $10 \mu \mathrm{L}$. (Video $S 2$ of a similar device at high speed $~ 120 x$ ). 


\section{Conclusion}

In conclusion we achieved a highly effective fluid flow manipulation method in $\mu$ PADs by intregrating cholinium-based crosslinked poly(ionic liquid) hydrogel pumps which have a high water retention capacity and thus excellent control on directing the fluid flow on the paper device. To our knowledge, these hydrogel negative pumps have the highest water retention capacity demonstrated in a $\mu$ PADs up to now, compared to similar negative passive pumps such as ionogels (50 $\pm 11 \mu \mathrm{L}$ of water for $30 \mu \mathrm{L}$ of ionogel [39]) or paper pumps, as the ones presented by Kokalj et al.[45] ( $17 \mu \mathrm{L}$ depending on the paper dimension).

These high capacity negative flow pumps open the possibility for sequential multi-step analysis enabling several different tests running in the same small-scale device owing to their high degree of water absorption and fluid control ability. Moreover, due to their influence on flow direction, they also open possibilities for developing $\mu$ PADs with no valves or external pumps. The biocompatibility of the choline-derived material should also open possibilities for point-of-care measurements, and potentially many other applications. This study demonstrates the feasibility of using cholinium - based poly(ionic liquid) hydrogels as a passive pump material for many future microfluidic applications.

\section{Acknowledgments}

The project was carried out with the support of the Ramón y Cajal programme (Ministerio de Economía y Competitividad). A.T., L.F., and D.D. are grateful for financial support from the Marie Curie Initial Training Network OrgBIO funded by the European Community's FP7 People Programme (Marie Curie ITN, GA607896). F.B.L., L.B.D. and D.D. also acknowledge funding from the European Union's Seventh Framework Programme (FP7) for Research, Technological Development and Demonstration under grant agreement no. 604241 (NAPES) and L.F. and D.D. acknowledge funding from Science Foundation Ireland (SFI) under the Insight Centre for Data Analytics initiative, Grant Number SFI/12/RC/2289. F.B.L. acknowledges the Gobierno Vasco, Dpto. Industria, Innovación, Comercio y Turismo under ELKARTEKKK-2016/00088 and the funding support from Gobierno de España, Ministerio de Economia y Competitividad, with Grant No. BIO2016-80417-P. FBL and TA personally acknowledge Marian M. de Pancorbo for letting 
them to use her laboratory facilities at UPV/EHU. Authors also acknowledge Adhesive Research for the donation of the PSA samples.

\section{References}

[1] C.T. Culbertson, T.G. Mickleburgh, S.A. Stewart-James, K.A. Sellens, M. Pressnall, Micro total analysis systems: fundamental advances and biological applications, Analytical chemistry, 86(2013) 95-118.

[2] M. Czugala, B. Ziolkowski, R. Byrne, D. Diamond, F. Benito-Lopez, Materials science: the key to revolutionary breakthroughs in micro-fluidic devices, SPIE NanoScience+ Engineering, International Society for Optics and Photonics2011, pp. 81070C-C-10.

[3] M.I. Mohammed, S. Haswell, I. Gibson, Lab-on-a-chip or Chip-in-a-lab: Challenges of Commercialization Lost in Translation, Procedia Technology, 20(2015) 54-9.

[4] E. Fu, T. Liang, P. Spicar-Mihalic, J. Houghtaling, S. Ramachandran, P. Yager, Two-dimensional paper network format that enables simple multistep assays for use in low-resource settings in the context of malaria antigen detection, Analytical chemistry, 84(2012) 4574-9.

[5] E. Livak-Dahl, I. Sinn, M. Burns, Microfluidic chemical analysis systems, Annual review of chemical and biomolecular engineering, 2(2011) 325-53.

[6] P. Lisowski, P.K. Zarzycki, Microfluidic paper-based analytical devices ( $\mu$ PADs) and micro total analysis systems ( $\mu$ TAS): development, applications and future trends, Chromatographia, 76(2013) 120114.

[7] C.R. Mace, R.N. Deraney, Manufacturing prototypes for paper-based diagnostic devices, Microfluidics and nanofluidics, 16(2014) 801-9.

[8] A. Böhm, F. Carstens, C. Trieb, S. Schabel, M. Biesalski, Engineering microfluidic papers: effect of fiber source and paper sheet properties on capillary-driven fluid flow, Microfluidics and nanofluidics, 16(2014) 789-99.

[9] Y. He, Y. Wu, J.-Z. Fu, W.-B. Wu, Fabrication of paper-based microfluidic analysis devices: a review, RSC Advances, 5(2015) 78109-27.

[10] C. Xu, L. Cai, M. Zhong, S. Zheng, Low-cost and rapid prototyping of microfluidic paper-based analytical devices by inkjet printing of permanent marker ink, Rsc Advances, 5(2015) 4770-3.

[11] S.-G. Jeong, S.-H. Lee, C.-H. Choi, J. Kim, C.-S. Lee, Toward instrument-free digital measurements: a three-dimensional microfluidic device fabricated in a single sheet of paper by double-sided printing and lamination, Lab on a Chip, 15(2015) 1188-94.

[12] R. Albatal, P. Mulhem, Y. Chiaramella, T.-J. Chin, Comparing image segmentation algorithms for content based image retrieval systems, Singaporean-French Symposiums SinFra 20092008, p. 66.

[13] A.W. Martinez, S.T. Phillips, Z. Nie, C.-M. Cheng, E. Carrilho, B.J. Wiley, et al., Programmable diagnostic devices made from paper and tape, Lab on a Chip, 10(2010) 2499-504. 
[14] E. Fu, B. Lutz, P. Kauffman, P. Yager, Controlled reagent transport in disposable 2D paper networks, Lab on a Chip, 10(2010) 918-20.

[15] J. Houghtaling, T. Liang, G. Thiessen, E. Fu, Dissolvable bridges for manipulating fluid volumes in paper networks, Analytical chemistry, 85(2013) 11201-4.

[16] B. Lutz, T. Liang, E. Fu, S. Ramachandran, P. Kauffman, P. Yager, Dissolvable fluidic time delays for programming multi-step assays in instrument-free paper diagnostics, Lab on a Chip, 13(2013) 2840-7.

[17] S. Jahanshahi-Anbuhi, A. Henry, V. Leung, C. Sicard, K. Pennings, R. Pelton, et al., Paper-based microfluidics with an erodible polymeric bridge giving controlled release and timed flow shutoff, Lab on a Chip, 14(2014) 229-36.

[18] B.J. Toley, J.A. Wang, M. Gupta, J.R. Buser, L.K. Lafleur, B.R. Lutz, et al., A versatile valving toolkit for automating fluidic operations in paper microfluidic devices, Lab on a Chip, 15(2015) 1432-44.

[19] R.R. Niedl, C. Beta, Hydrogel-driven paper-based microfluidics, Lab on a Chip, 15(2015) 2452-9.

[20] X. Wei, T. Tian, S. Jia, Z. Zhu, Y. Ma, J. Sun, et al., Target-responsive DNA hydrogel mediated "stopflow" microfluidic paper-based analytic device for rapid, portable and visual detection of multiple targets, Analytical chemistry, 87(2015) 4275-82.

[21] B. Ziółkowski, L. Florea, J. Theobald, F. Benito-Lopez, D. Diamond, Self-protonating spiropyran-coNIPAM-co-acrylic acid hydrogel photoactuators, Soft Matter, 9(2013) 8754-60.

[22] S.-k. Ahn, R.M. Kasi, S.-C. Kim, N. Sharma, Y. Zhou, Stimuli-responsive polymer gels, Soft Matter, 4(2008) 1151-7.

[23] A. Drozdov, C.-G. Sanporean, Modeling the effects of temperature and pH on swelling of stimuliresponsive gels, European Polymer Journal, 73(2015) 278-96.

[24] M. Irie, Stimuli-responsive poly (N-isopropylacrylamide). Photo-and chemical-induced phase transitions, Responsive Gels: Volume Transitions II, (1993) 49-65.

[25] R. Po, Water-absorbent polymers: a patent survey, Journal of Macromolecular Science, Part C: Polymer Reviews, 34(1994) 607-62.

[26] H. Suzuki, Stimulus-responsive gels: Promising materials for the construction of micro actuators and sensors, Journal of intelligent material systems and structures, 17(2006) 1091-7.

[27] S. Sugiura, K. Sumaru, K. Ohi, K. Hiroki, T. Takagi, T. Kanamori, Photoresponsive polymer gel microvalves controlled by local light irradiation, Sensors and Actuators A: Physical, 140(2007) 176-84.

[28] B. Ziółkowski, M. Czugala, D. Diamond, Integrating stimulus responsive materials and microfluidics: The key to next-generation chemical sensors, Journal of Intelligent Material Systems and Structures, 24(2013) 2221-38.

[29] A. Tudor, J. Saez, L. Florea, F. Benito-Lopez, D. Diamond, Poly(ionic liquid) thermo-responsive hydrogel microfluidic actuators, Sensors and Actuators B: Chemical, 247(2017) 749-755.

[30] C. Delaney, P. McCluskey, S. Coleman, J. Whyte, N. Kent, D. Diamond, Precision control of flow rate in microfluidic channels using photoresponsive soft polymer actuators, Lab on a Chip, 17(2017) 20132021. 
[31] S. Coleman, J. Schiphorst, A. B. Azouz, S. Bakker, A. P. H. J. Schenning, D. Diamond, Tuning microfluidic flow by pulsed light oscillating spiropyran-based polymer hydrogel valves, Sensors and Actuators B: Chemical, 245(2017), 81-86.

[32] R. Byrne, F. Benito-Lopez, D. Diamond, Materials science and the sensor revolution, Materials Today, 13(2010) 16-23.

[33] F. Benito-Lopez, R. Byrne, A.M. Răduţă, N.E. Vrana, G. McGuinness, D. Diamond, Ionogel-based light-actuated valves for controlling liquid flow in micro-fluidic manifolds, Lab on a Chip, 10(2010) 195201.

[34] F. Benito-Lopez, M. Antoñana-Díez, V.F. Curto, D. Diamond, V. Castro-López, Modular microfluidic valve structures based on reversible thermoresponsive ionogel actuators, Lab on a Chip, 14(2014) 3530-8.

[35] M. Czugala, C. O’Connell, C. Blin, P. Fischer, K.J. Fraser, F. Benito-Lopez, et al., Swelling and shrinking behaviour of photoresponsive phosphonium-based ionogel microstructures, Sensors and Actuators B: Chemical, 194(2014) 105-13.

[36] J. Le Bideau, L. Viau, A. Vioux, Ionogels, ionic liquid based hybrid materials, Chemical Society Reviews, 40(2011) 907-25.

[37] A. Kavanagh, R. Byrne, D. Diamond, K.J. Fraser, Stimuli Responsive Ionogels for Sensing Applications-An Overview, Membranes, 2(2012) 16-39.

[38] T. Akyazi, J. Saez, J. Elizalde, F. Benito-Lopez, Fluidic flow delay by ionogel passive pumps in microfluidic paper-based analytical devices, Sensors and Actuators B: Chemical, 233(2016) 402-8.

[39] T. Akyazi, N. Gil-González, L. Basabe-Desmonts, E. Castaño, M. Morant-Miñana, F. Benito-Lopez, Manipulation of fluid flow direction in microfluidic paper-based analytical devices with an ionogel negative passive pump, Sensors and Actuators B: Chemical, 247(2017) 114-23.

[40] M. Isik, T. Lonjaret, H. Sardon, R. Marcilla, T. Herve, G.G. Malliaras, et al., Cholinium-based ion gels as solid electrolytes for long-term cutaneous electrophysiology, Journal of Materials Chemistry C, 3(2015) 8942-8.

[41] M. Isik, H. Sardon, M. Saenz, D. Mecerreyes, New amphiphilic block copolymers from lactic acid and cholinium building units, RSC Advances, 4(2014) 53407-10.

[42] D. Mecerreyes, Polymeric ionic liquids: Broadening the properties and applications of polyelectrolytes, Progress in Polymer Science, 36(2011) 1629-48.

[43] J. Yuan, M. Antonietti, Poly (ionic liquid) s: polymers expanding classical property profiles, Polymer, 52(2011) 1469-82.

[44] J. Yuan, D. Mecerreyes, M. Antonietti, Poly (ionic liquid) s: an update, Progress in Polymer Science, 38(2013) 1009-36.

[45] T. Kokalj, Y. Park, M. Vencelj, M. Jenko, L. P. Lee, Self-powered Imbibing Microfluidic Pump by Liquid Encapsulation: SIMPLE, Lab on a Chip, 14(22) 4329-33. 
